\title{
Pulse Compression with Large-Mode-Area Photonic Crystal Fibres
}

\author{
Péter Dombi \\ Wigner Research Centre for Physics, 1121 Budapest, Hungary \\ phone: +361392 2209, e-mail:dombi.peter@wigner.mta.hu
}

\begin{abstract}
Photonic crystal fibres (PCFs) are established tools for supercontinuum generation and femtosecond pulse compression down to the few-femtosecond regime. With the advent of femtosecond oscillators delivering ever higher pulse energy, one needs a pulse energy scalable technique to achieve these goals. Therefore, I will review the usage of large-mode-area PCFs for femtosecond applications and introduce some related new nonlinear fiber phenomena.
\end{abstract}

Keywords: photonic crystal fibres, microstructured fibres, femtosecond pulse compression, nonlinear fibre optics.

\section{FEMTOSECOND PULSE COMPRESSION CONSIDERATIONS}

Nonlinear optical phenomena such as self phase modulation, four wave mixing and Raman processes in various fiber and bulk media can be efficiently exploited for the compression of femtosecond pulses. These techniques are particularly important in few-cycle and single-cycle laser pulse generation [1-3] and other cases where shorter pulses are required than the gain and/or dispersion bandwidth allowed by laser oscillator or amplifier architectures. Therefore, one has to perform extra cavity pulse compression which necessitates (i) nonlinearities to increase the spectral content of the pulse and (ii) proper spectral phase management in order to end up with a pulse which is as close to the transform limit as possible. In some cases, elements (i) and (ii) are realized in the same medium making use of the strong interplay of linear and nonlinear phenomena during the compression process. Examples include self-compression in filaments [4] or in the soliton regime.

For most input pulses, however, more robust and broadband solutions are offered by schemes where the spectral broadening and pulse compression stages are separated [5-7]. In these cases, the spectral broadening stage usually consists of single-mode solid-core, multi-mode, rod-type or gas-filled hollow-core fibers. The spectrum of femtosecond oscillator pulses with up to $\sim 30 \mathrm{~nJ}$ energy can be efficiently broadened in short single-mode glass fibers with $2-5 \mu \mathrm{m}$ core diameter [5] whereas the $\sim \mathrm{mJ}$ pulses of femtosecond amplifiers can be efficiently guided and broadened in gas-filled capillaries [7]. With the recent advent of femtosecond technology resulting in high-energy, $100 \mathrm{~nJ}$...several $\mu \mathrm{J}$ pulses with $>1 \mathrm{MHz}$ repetition rate [8-10] (in the Ti:sapphire case, directly from the oscillator), new methods for pulse compression have to be devised for these novel parameter regimes. This has to take into account the desired spectral broadening and the damage threshold of nonlinear optical elements. A particular challenge for Ti:sapphire oscillators is also represented by the large bandwidth of both the source and the required spectral broadening, therefore, schemes devised for the compression of multi-100-fs pulses can not be implemented.

\section{PULSE COMPRESSION WITH LARGE-MODE-AREA FIBERS}

Large mode area (LMA) photonic crystal fibers (PCFs) offer endlessly single mode guiding combined with a mode field diameter of up to around $100 \mu \mathrm{m}$ and an $\mathrm{M}^{2}$ value of $1.05-1.2$ at the fiber output. These features make them suitable for high-energy pulse compression at $\mathrm{MHz}$ repetition rates. The large mode area prohibits optical damage at the fiber entrance and fiber guiding allows sufficient nonlinearity for pulse compression at the same time, as indicated by supercontinuum (SC) generation experiments. The potential offered by LMA PCFs was shown recently in compression experiments with relatively long and/or heavily chirped pulses [11], based on the principle of chirped-pulse supercontinuum generation [12]. It was also possible to perform efficient and energy scalable compression with transform limited input [13]. A particularly interesting nonlinear fibre optical phenomenon in this respect is the conversion of the chirp of the input pulses upon propagation in the fibre that we observed recently [14].

\section{CONCLUSIONS}

In conclusion, novel nonlinear fiber optics phenomena can be exploited to efficiently perform femtosecond pulse compression in novel parameter regimes corresponding to the operation of long-cavity femtosecond oscillators, disk lasers, fibre amplifiers etc. Large-mode-area photonic crystal fibres offer a scalable alternative for this purpose and these developments also open new pathways for applications of these light sources including timeresolved plasmonics [15], high harmonic generation with high repetition rates, ultrafast spectroscopy, ultrafast photocathode development and many more. 


\section{ACKNOWLEDGEMENTS}

I acknowledge support from the Bolyai Fellowship of the Hungarian Academy of Sciences and from the Hungarian Scientific Research Fund (OTKA Project 109257).

\section{REFERENCES}

[1] P. Dombi et al., Opt. Express 13, 10888 (2005) and references therein.

[2] E. Matsubara, K. Yamane, T. Sekikawa, M. Yamashita: J. Opt. Soc. Am. B 24, 985 (2007).

[3] G. Krauss, S. Lohss, T. Hanke, A. Sell, S. Eggert, R. Huber, A. Leitenstorfer: Nature Phot. 4, 33 (2010).

[4] G. Stibenz, N. Zhavoronkov, G. Steinmeyer: Opt. Lett. 31, 274-276 (2006).

[5] P. Dombi et al.: New J. Phys. 6, 39 (2004).

[6] C. Jocher, T. Eidam, S. Haedrich, J. Limpert, and A. Tünnermann: Opt. Letters 37, 4407-4409 (2012).

[7] M. Nisoli, S. De Silvestri, O. Svelto: Appl. Phys. Letters 68, 2793 (1996).

[8] S. Naumov, A. Fernandez, R. Graf, P. Dombi, F. Krausz, A. Apolonski: New J. Phys. 7, 216 (2005).

[9] P. Dombi, P. Antal: Laser Phys. Lett. 4, 538 (2007).

[10] T. Südmeyer et al.: Nature Phot. 2, 599 (2008).

[11] T. Ganz, V. Pervak, A. Apolonski, P. Baum: Opt. Lett. 36, 1107 (2011).

[12] P. Dombi, P. Antal, J. Fekete, R. Szipöcs, Z. Várallyay: Appl. Phys. B 88, 379 (2007).

[13] J. Fekete, P. Rácz and P. Dombi: Appl. Phys. B 111, 415-418 (2013).

[14] P. Dombi, P. Rácz, L. Veisz and P. Baum: Opt. Lett. 39, 2232-2235 (2014).

[15] P. Rácz et al.: Appl. Phys. Lett. 98, 111116 (2011). 